\title{
Clinical Characteristics of Pesticide Self-Harm as Associated with Suicide Attempt Repetition Status
}

This article was published in the following Dove Press journal:

Neuropsychiatric Disease and Treatment

\author{
Wei-Chen Huang (ID) \\ Tzung-Hai Yen $\mathbb{D}^{2,3}$ \\ Long $\operatorname{Lin}^{4}$ \\ Chemin Lin iD $^{3,5,6}$ \\ Yeong-Yuh Juang ${ }^{7}$ \\ Bi-Hwa Wang ${ }^{8}$ \\ Shwu-Hua Lee ${ }^{1,3}$ \\ 'Department of Psychiatry, Chang Gung \\ Memorial Hospital, Linkou, Taiwan; \\ ${ }^{2}$ Department of Nephrology and Clinical \\ Poison Center, Chang Gung Memorial \\ Hospital, Linkou, Taiwan; ${ }^{3}$ School of \\ Medicine, College of Medicine, Chang \\ Gung University, Taoyuan, Taiwan; \\ ${ }^{4}$ School of Traditional Chinese Medicine, \\ College of Medicine, Chang Gung \\ University, Taoyuan, Taiwan; \\ ${ }^{5}$ Department of Psychiatry, Chang Gung \\ Memorial Hospital, Keelung, Taiwan; \\ ${ }^{6}$ Community Medicine Research Center, \\ Chang Gung Memorial Hospital, Keelung, \\ Taiwan; ${ }^{7}$ Department of Psychiatry, Koo \\ Foundation Sun Yat-Sen Cancer Center, \\ Taipei, Taiwan; ${ }^{8}$ School of Nursing, \\ College of Medicine, Chang Gung \\ University, Taoyuan, Taiwan
}

Correspondence: Shwu-Hua Lee Department of Psychiatry, Chang Gung Memorial Hospital, 5 Fu-Shin Road, KweiShan, 333, Linkou, Taiwan

Tel +88633281200 Ext 2439

Fax +886-3-3280267

Email shlee@cgmh.org.tw
Purpose: Previous suicide behavior predicts future suicide risk. Studies of suicide repetition are important for suicide prevention. This study examined the clinical characteristics and psychiatric comorbidities of pesticide self-harm as associated with suicide attempt repetition status.

Patients and Methods: This retrospective, observational study included patients admitted to a medical center in northern Taiwan between 2000 and 2015 following suicide attempts by pesticide. Diagnoses were made by a consultation-liaison psychiatry team based on the DSM IV-TR criteria. Independent samples $t$-test was used to analyze the quantitative variables and Pearson's Chi-squared test with a Bonferroni adjustment for categorical variables. Univariate binary logistic regression analysis was conducted to determine the predictors for repeated suicide attempt and multivariate binary logistic regression analysis to identify significant associated factors.

Results: Among 151 patients, organophosphate pesticides were the most used (80.8\%). The average age was 52.9 \pm 17.2 years. Most patients were married males from rural areas, with depressive disorder most often diagnosed (40.4\%). Nearly one-third of them were suicide repeaters $(n=43,28.5 \%)$; these patients were less likely to be married $(53.5 \%, p=0.001)$, had previously used psychiatric services $(72.1 \%, \mathrm{p}<0.001)$ and chose complex suicide $(46.5 \%$, $\mathrm{p}=0.014$ ) more often compared to nonrepeaters. No significant differences were found between repeaters and nonrepeaters regarding psychiatric comorbidities. Multivariate logistic regression analysis revealed that marital status (odds ratio: $2.916,95 \%$ confidence interval: 1.234-6.891, $\mathrm{p}=0.015$ ) and previous psychiatric services usage (odds ratio: $6.897,95 \%$ confidence interval: $3.012-15.625, \mathrm{p}<0.001)$ were significant risk factors associated with suicide repetition.

Conclusion: Pesticide suicide repeaters were characterized by less likely to be married and more likely to use psychiatric services before suicide attempts. These features help ensuring that the needs of people who have made a suicide attempt are addressed, and it reduces the likelihood of repetition.

Keywords: self-harm behavior, self-poisoning, intentional poisoning, organophosphate

\section{Introduction}

Approximately 800,000 people die by suicide every year (World Health Organization, 2020). Although previous meta-analysis failed to distinguish patients with a high suicide risk group, ${ }^{1}$ knowing the characteristics of suicide repeaters can still help psychiatrists providing a patient-centred care, ensuring that the needs of people who have made a suicide attempt are addressed. A systemic review analyzing repeaters by different suicide means revealed characteristics of unemployment, unmarried status, psychiatric illness. ${ }^{2}$ While focusing on the study of repeaters by 
self-poisoning, they were female predominant and having previous psychiatric treatment, they tend to choose antidepressants overdose. ${ }^{3}$ Another study showed no gender significance between repeaters and nonrepeaters groups, yet alcohol use disorder was seen more in male repeaters. ${ }^{4}$

Pesticide self-poisoning is one of the most used methods of suicide globally. Although there has been a decline in lethal pesticide self-poisoning in recent years, pesticide self-poisoning remains an important public health issue, accounting for approximately $14 \%$ of suicide death worldwide. ${ }^{5}$ According to a systemic review conducted by Gunnel et al, ${ }^{6}$ national bans on highly lethal pesticides are effective in reducing overall suicide mortality rates, the reducing trend was also observed in Taiwan. Pesticides with a lower toxicity are still dangerous due to their easy accessibility, in a study conducted by Lin et $\mathrm{al}^{7}$ the mortality rate of organophosphate exposure, which is the most used insecticide throughout the world and is widely used in Taiwan, could be as high as $12.71 \%$, once patients are in respiratory failure and are mechanically ventilated, the mortality rate can rise to $50 \% .^{8}$ In a study by Sahin et $\mathrm{al}^{9}{ }^{9}$ suicide attempters who chose organophosphates were female predominant, $51.8 \%$ were unmarried, they had a mean age of $22.1 \pm 9.2$ years, and $76.8 \%$ of them were younger than age 24 .

There is a paucity of studies in the literature examining psychiatric comorbidities or suicide repetition in patients who have attempted suicide by pesticide. In a review article, Carrol et al reported that fewer Asian patients attempted suicide by pesticide at risk of repetition comparing to European patients, ${ }^{10}$ nevertheless, a 2019 prospective cohort study in Sri Lanka showed $48.7 \%$ of suicide repeaters by pesticide chose pesticide again in repeated attempts. ${ }^{11}$ In a study of 39 Fiji patients who attempted suicide mostly by drug and pesticide ingestion, Aghanwa ${ }^{12}$ reported that social problems and psychiatric comorbidities were noted in $64.1 \%$ of cases. Our previous study ${ }^{13}$ of 157 Taiwanese patients who attempted suicide by paraquat ingestion revealed that mood disorders $(54.0 \%)$ were the most common psychiatric comorbidities.

To our knowledge, a study of suicide repeaters by pesticide is lacking. Therefore, the objective of this retrospective, observational study was to examine the clinical characteristics and psychiatric comorbidities of patients who attempted suicide by organophosphates, carbamates or glyphosates and analyze the variations in these parameters between repeaters and nonrepeaters.

\section{Patients and Methods}

\section{Ethical Statements}

This retrospective, observational study followed the Declaration of Helsinki and was approved by the Medical Ethics Committee of Chang Gung Memorial Hospital, Taiwan (Institutional Review Board number 201800245B0). The IRB waived the need for specific informed consent from the patients because this was a retrospective study based on case research or cases treated or diagnosed by clinical routines without enrollment of HIV positive cases. The confidentiality of each patient's data was kept.

\section{Patients}

\section{Data Collection from Clinical Records}

Between January 1, 2000, and December 31, 2015, a total of 151 patients who attempted suicide by pesticide received a consultation-liaison psychiatry evaluation at Chang Gung Memorial Hospital (CGMH), Linkou, Taiwan. At CGMH, it is routine clinical practice for patients who attempt suicide to have a formal consultationliaison psychiatry evaluation. All patients who attempted suicide by pesticide and received a complete consultationliaison psychiatry evaluation at CGMH were analyzed. We reviewed the medical records, which included emergency department records, admission, progress and discharge records, nursing records, psychiatric consultation records, and psychiatric outpatient clinical records. After reviewing all records, psychiatric diagnosis was made using the DSM IV-TR criteria. Demographic data such as age, gender, and job were recorded, along with the patients` systemic disease and adverse outcome of pesticide poisoning, including intubation or aspiration pneumonia, but excluding death. All laboratory findings available in the aforementioned medical records were documented, which included serum acetylcholinesterase (AChE) levels (reference level: 7000-19,000 U/L) during the hospital stay (both emergency department and ward admission), and we only recorded the nadir AChE level. The clinical courses of organophosphate and carbamate intoxication include acute cholinergic crisis following cholinesterase depression, intermediate syndrome with unclear mechanism, and delayed polyneuropathy caused by the suppression of neuropathy target esterase. ${ }^{14}$ Therefore, the nadir serum $\mathrm{AChE}$ levels have been used as a biomarker of acute cholinergic crisis. The pesticide brand used for suicide was recorded in the medical record, and we categorized them into three groups (organophosphate, carbamate, 
glyphosate) according to the 2018 Taiwan official pesticide guideline. The reason for choosing organophosphate, carbamate and glyphosate is they are the top three most heavily used pesticides for self-poisoning in Taiwan. ${ }^{15}$ When suicide attempters used two or more means of suicide or used two or more toxins in ingestion, it was recorded as a complex suicide. ${ }^{16}$

\section{Detoxification Protocols}

Organophosphate and carbamate patients were treated with gastric lavage with $2 \mathrm{~L}$ of normal saline, followed by $1 \mathrm{~g} /$ $\mathrm{kg}$ activated charcoal and $250 \mathrm{~mL}$ magnesium citrate infused via nasogastric tube. ${ }^{17}$ Magnesium citrate was used to prevent constipation after charcoal therapy. Since all organophosphate and carbamate patients had depressed serum cholinesterase levels, they were treated with antidotes that included anticholinergic and oxime drugs. Intravenous atropine was initiated at $2 \mathrm{mg}$ every 1-2 hours and titrated to the resolution of bronchial secretions and bronchospasm. Pralidoxime therapy ( $1 \mathrm{~g}$ every 4 hours, intravenous) was also prescribed to patients with cholinergic crisis. Glyphosate patients were intensively treated by gastric lavage with a large amount of normal saline followed by active charcoal and magnesium citrate administration. There is no specific antidote available for glyphosate poisoning, and its management is mainly symptomatic and supportive.

\section{Statistical Analysis}

Continuous variables were documented as the means and standard deviations, and categorical variables were documented as numbers and percentages. All medical factors related to mortality and morbidity were selected by the nephrologist based on clinical and existing knowledge about organophosphate intoxication. We used SPSS version 22 (Statistical Product and Service Solutions) to perform all statistical analyses. We used Pearson's Chi-squared test with a Bonferroni adjustment and independent samples $t$-test to assess associations as appropriate. Univariate binary logistic regression analysis was performed to analyze the predictors for repeat suicide attempt. To control for confounding factors, multivariate binary logistic regression analysis was performed to analyze the significant predictors after univariate analysis. An effect size with its corresponding 95\% confidence interval (CI) was calculated for significant associations. The $p$-values were recorded as twosided, and the significance level was set at 0.05 .

\section{Results}

\section{Baseline Demographics}

Race-ethnicity of our all patients was Taiwanese. As shown in Table 1, organophosphates were the most commonly used pesticide $(80.8 \%)$, followed by glyphosates $(10.6 \%)$ and carbamates (8.6\%). The mean age of the patients was $52.9 \pm 17.2$ years and most were male (69.5\%) and married (72.2\%). Although $96.7 \%$ of patients had a past psychiatric diagnosis, only $38.4 \%$ had used psychiatric services, and $15.2 \%$ had used our psychiatric outpatient clinic before this suicide attempt. One-third of the patients (31.8\%) attempted complex suicide. Most of the patients (79.5\%) were from rural areas, and $45 \%$ of the patients were unemployed. Some patients suffered from a chronic systemic disease, including hypertension (23.8\%), diabetes mellitus $(9.9 \%)$, and hepatic disease $(9.3 \%)$.

A total of 43 (28.5\%) patients reported a previous suicide attempt and were classified as suicide repeaters (repeaters), while the other 108 (71.5\%) patients were classified as suicide nonrepeaters (nonrepeaters). Repeaters were less likely to be married $(53.5 \%, \mathrm{p}=0.001)$, previously used psychiatric services $(72.1 \%, \mathrm{p}<0.001)$, and chose complex suicide $(46.5 \%$, $\mathrm{p}=0.014)$ more often compared to nonrepeaters. In Table 2, it showed 54.2\% who chose complex suicide applied alcohol intoxication, $27 \%$ applied illicit drug or other medication overdose. In addition, there were some trends among repeaters and nonrepeaters, although there were no significant differences, including age (repeaters $49.3 \pm 15.2$ years, nonrepeaters $54.3 \pm 17.8$ years, $\mathrm{p}=0.104$ ), rate of visiting our psychiatric outpatient clinic before this suicide attempt (repeaters 23.3\%, nonrepeaters $12 \%, \mathrm{p}=0.083$ ), and time elapsed between poisoning and hospital arrival (repeaters $354.0 \pm 389.6$ minutes, nonrepeaters $245.4 \pm 238.3$ minutes, $\mathrm{p}=0.055$ ).

\section{Clinical Manifestations and Laboratory Findings}

Table 3 shows that pesticide intoxication produces many serious systemic complications, including acute respiratory failure (49.7\%), aspiration pneumonia (39.7\%), sepsis (3.3\%), seizure (3.3\%), hypoxic encephalopathy $(3.3 \%)$, and gastrointestinal bleeding (3.3\%). The mean hospitalization duration was $18.6 \pm 15.2$ days. After discharge, $49.7 \%$ of the patients were regularly followed at our psychiatric outpatient clinic, and the mean follow-up period was 998.7 \pm 1561.4 days. Overall, there were no significant differences in the clinical manifestations and laboratory findings between repeaters and nonrepeaters (Tables 3 and 4), although some trends could be observed, including the 
Table I Baseline Demographics of Patients with Pesticide Poisoning, Stratified into Two Subgroups According to Suicide History as Repeaters and Nonrepeaters $(n=|5|)$

\begin{tabular}{|c|c|c|c|c|c|c|}
\hline \multirow[t]{2}{*}{ Variables } & $\begin{array}{l}\text { All Patients } \\
(n=15 I)\end{array}$ & $\begin{array}{l}\text { Repeaters } \\
(n=43)\end{array}$ & $\begin{array}{l}\text { Nonrepeaters } \\
(n=108)\end{array}$ & $x^{2}$ & df & P-value \\
\hline & n (\%) & n (\%) & n (\%) & & & \\
\hline Age, year (mean $\pm S D)$ & $52.9 \pm 17.2$ & $49.3 \pm 15.2$ & $54.3 \pm 17.8$ & & & 0.104 \\
\hline Male & $105(69.5)$ & $31(72.1)$ & $74(68.5)$ & 0.186 & I & 0.667 \\
\hline $\begin{array}{l}\text { Marital status } \\
\text { Not married } \\
\text { Married }\end{array}$ & $\begin{array}{l}42(27.8) \\
109(72.2)\end{array}$ & $\begin{array}{l}20(46.5) \\
23(53.5)\end{array}$ & $\begin{array}{l}22(20.4) \\
86(79.6)\end{array}$ & 10.468 & I & $0.00 I^{* *}$ \\
\hline $\begin{array}{l}\text { Pesticide } \\
\text { Organophosphate } \\
\text { Carbamate } \\
\text { Glyphosate }\end{array}$ & $\begin{array}{l}122(80.8) \\
13(8.6) \\
16(10.6)\end{array}$ & $\begin{array}{l}33(76.7) \\
2(4.7) \\
8(18.6)\end{array}$ & $\begin{array}{l}89(82.4) \\
11(10.2) \\
8(7.4)\end{array}$ & 4.855 & 2 & 0.088 \\
\hline $\begin{array}{l}\text { Season of the year } \\
\text { Spring } \\
\text { Summer } \\
\text { Autumn } \\
\text { Winter }\end{array}$ & $\begin{array}{l}23(15.2) \\
42(27.8) \\
46(30.5) \\
40(26.5)\end{array}$ & $\begin{array}{l}7(16.3) \\
11(25.6) \\
15(34.9) \\
10(23.3)\end{array}$ & $\begin{array}{l}16(14.8) \\
31(28.7) \\
31 \\
30(28.7) \\
30\end{array}$ & 0.774 & 3 & 0.856 \\
\hline Past psychiatric service usage & $58(38.4)$ & $31(72.1)$ & $27(25.0)$ & 28.832 & I & $0.000 * * *$ \\
\hline $\begin{array}{l}\text { Visit our psychiatric outpatient clinic before attempting } \\
\text { suicide }\end{array}$ & $23(15.2)$ & $10(23.3)$ & $13(12.0)$ & 2.998 & I & 0.083 \\
\hline Complex suicide & $48(31.8)$ & $20(46.5)$ & $28(25.9)$ & 6.011 & I & $0.014 *$ \\
\hline Unemployed & $68(45.0)$ & $18(4 \mid .9)$ & $50(46.3)$ & 0.244 & I & 0.621 \\
\hline $\begin{array}{l}\text { Residence } \\
\text { Rural area } \\
\text { Urban area }\end{array}$ & $\begin{array}{l}120(79.5) \\
31(20.5)\end{array}$ & $\begin{array}{l}36(83.7) \\
7(16.3)\end{array}$ & $\begin{array}{l}84(77.8) \\
24(22.2)\end{array}$ & 0.666 & I & 0.415 \\
\hline Living alone & II (7.3) & $4(9.3)$ & $7(6.5)$ & 0.362 & I & 0.547 \\
\hline Transferred from other hospital & $97(64.2)$ & $25(58.1)$ & $72(66.7)$ & 0.973 & I & 0.324 \\
\hline $\begin{array}{l}\text { Time elapsed between poisoning and hospital } \\
\text { arrival, minute (mean } \pm S D)\end{array}$ & $277.1 \pm 293.3$ & $354.0 \pm 389.6$ & $245.4 \pm 238.3$ & & & 0.055 \\
\hline Psychiatric diagnosis & I 46 (96.7) & $42(97.7)$ & $104(96.3)$ & 0.182 & I & 0.669 \\
\hline Diabetes mellitus & $15(9.9)$ & $2(4.7)$ & $13(12.0)$ & $\mathrm{I} .875$ & I & 0.171 \\
\hline Hypertension & $36(23.8)$ & $8(18.6)$ & $28(25.9)$ & 0.908 & I & 0.341 \\
\hline Coronary artery disease & $5(3.3)$ & $0(0.0)$ & $5(4.6)$ & 2.059 & I & 0.151 \\
\hline Cerebrovascular accident & $8(5.3)$ & $2(4.7)$ & $6(5.6)$ & 0.050 & I & 0.823 \\
\hline Thyroid disease & $3(2.0)$ & $0(0.0)$ & $3(2.8)$ & 1.219 & I & 0.270 \\
\hline Rheumatic disease & $8(5.3)$ & $\mathrm{I}(2.3)$ & $7(6.5)$ & 1.059 & I & 0.304 \\
\hline Cancer & $6(4.0)$ & $\mathrm{I}(2.3)$ & $5(4.6)$ & 0.428 & I & 0.513 \\
\hline
\end{tabular}

(Continued) 
Table I (Continued).

\begin{tabular}{|c|c|c|c|c|c|c|}
\hline \multirow[t]{2}{*}{ Variables } & $\begin{array}{l}\text { All Patients } \\
(n=I 5 I)\end{array}$ & $\begin{array}{l}\text { Repeaters } \\
(n=43)\end{array}$ & $\begin{array}{l}\text { Nonrepeaters } \\
(n=108)\end{array}$ & $x^{2}$ & df & P-value \\
\hline & n (\%) & n (\%) & n (\%) & & & \\
\hline Asthma & $3(2.0)$ & $2(4.7)$ & I (0.9) & 2.192 & I & 0.139 \\
\hline Cardiovascular disease & $9(6.0)$ & I (2.3) & $8(7.4)$ & 1.417 & I & 0.234 \\
\hline Hepatic disease & $14(9.3)$ & $5(11.6)$ & $9(8.3)$ & 0.397 & I & 0.529 \\
\hline Pulmonary disease & $5(3.3)$ & I (2.3) & $4(3.7)$ & 0.182 & I & 0.669 \\
\hline Renal disease & $3(2.0)$ & I (2.3) & $2(1.9)$ & 0.035 & I & 0.851 \\
\hline Neurological disease & $7(4.6)$ & I (2.3) & $6(5.6)$ & 0.726 & I & 0.394 \\
\hline Gastrointestinal disease & $13(8.6)$ & $4(9.3)$ & $9(8.3)$ & 0.037 & I & 0.848 \\
\hline Anemia & $105(69.5)$ & $31(72.1)$ & $74(68.5)$ & 0.935 & I & 0.334 \\
\hline
\end{tabular}

Notes: ${ }^{*}<<0.05, * * p<0.01,{ }^{*} * *_{p}<0.001$.

follow-up rate at our psychiatric outpatient clinic after discharge (repeaters $58.1 \%$, nonrepeaters $46.3 \%, \mathrm{p}=0.189$ ) and the follow-up duration at our psychiatric outpatient clinic after discharge (repeaters $780.4 \pm 1470.9$ days, nonrepeaters $1103.3 \pm 1607.5$ days, $p=0.419$ ), serum AChE level (repeaters $3058.6 \pm 5140.2 \mathrm{U} / \mathrm{L}$, nonrepeaters $1897.8 \pm 2870.2 \mathrm{U} / \mathrm{L}$, $\mathrm{p}=0.087$ ).

\section{Predictors for Repeated Suicide Attempt}

As shown in Table 5, multivariate logistic regression analysis revealed that marital status (odds ratio: 2.916, 95\% confidence interval: $1.234-6.891, \mathrm{p}=0.015)$ and previous

Table 2 Methods Used in Complex Suicide of Patients with Pesticide Poisoning, Stratified into Two Subgroups According to Suicide History as Repeaters and Nonrepeaters $(n=48)$

\begin{tabular}{|c|c|c|c|}
\hline \multirow[t]{2}{*}{ Variables } & $\begin{array}{l}\text { All Patients } \\
(n=48)\end{array}$ & $\begin{array}{l}\text { Repeaters } \\
(n=20)\end{array}$ & $\begin{array}{l}\text { Nonrepeaters } \\
(n=28)\end{array}$ \\
\hline & n (\%) & n (\%) & n (\%) \\
\hline Alcohol & $26(54.2)$ & II (55.0) & $15(53.6)$ \\
\hline Illicit drugs & $5(10.4)$ & $2(10.0)$ & $3(10.7)$ \\
\hline Wrist cutting & $6(12.5)$ & $3(15.0)$ & $3(10.7)$ \\
\hline $\begin{array}{l}\text { Charcoal } \\
\text { burning }\end{array}$ & I (2.I) & $0(0.0)$ & I (3.6) \\
\hline $\begin{array}{l}\text { Drug } \\
\text { overdose }\end{array}$ & $9(18.8)$ & $3(15.0)$ & $6(21.4)$ \\
\hline $\begin{array}{l}\text { Drinking } \\
\text { detergent }\end{array}$ & $2(4.2)$ & I (5.0) & I (3.6) \\
\hline $\begin{array}{l}\text { Stabbing } \\
\text { oneself }\end{array}$ & $2(4.2)$ & $0(0.0)$ & $2(7.1)$ \\
\hline
\end{tabular}

psychiatric services usage (odds ratio: $6.897,95 \%$ confidence interval: $3.012-15.625, \mathrm{p}<0.001)$ were significant risk factors associated with repeated suicide attempt.

\section{Psychiatric Comorbidities}

Psychiatric comorbidities are listed in Table 6. Psychiatry consultation-liaison evaluations revealed that depressive disorder, substance use disorder and adjustment disorder were present in $66.9 \%, 30.5 \%$ and $15.2 \%$ of cases, respectively. In addition, among all substance users, $84.8 \%$ had an alcohol use disorder. For psychiatric comorbidities, there were no significant differences between repeaters and nonrepeaters, but some trends could be observed, including adjustment disorder (repeaters 7.0\%, nonrepeaters 18.5\%) and alcohol use disorder (repeaters $34.9 \%$, nonrepeaters $22.2 \%$ ).

\section{Discussion}

This study analyzed the characteristics of 151 patients who attempted suicide by pesticide and presented to hospital. Of these, $28.5 \%$ reported a previous suicide attempt (suicide repeaters). Half of the repeaters were not married, and they used more psychiatric services before this admission, which may indicate that if repeaters could be identified earlier, suicide repetition may be preventable.

Our study revealed $80.8 \%$ of suicide attempters used organophosphate as their suicide toxin, it reflected that organophosphate is commonly used pesticide in Taiwan. In a retrospective study that included 47 patients, $74.4 \%$ had respiratory failure, ${ }^{18}$ compared to $51.6 \%$ in our group, this may indicate the severity of our poisoning might be 
Table 3 Clinical Manifestations of Patients with Pesticide Poisoning, Stratified into Two Subgroups According to Suicide History as Repeaters and Nonrepeaters $(n=|5|)$

\begin{tabular}{|c|c|c|c|c|c|c|}
\hline \multirow[t]{2}{*}{ Variables } & $\begin{array}{l}\text { All Patients } \\
(n=|5|)\end{array}$ & $\begin{array}{l}\text { Repeaters } \\
(n=43)\end{array}$ & $\begin{array}{l}\text { Nonrepeaters } \\
(n=108)\end{array}$ & $x^{2}$ & df & P-value \\
\hline & n (\%) & n (\%) & n (\%) & & & \\
\hline Acute respiratory failure & $75(49.7)$ & $21(48.8)$ & $54(50.0)$ & 0.017 & 1 & 0.897 \\
\hline Aspiration pneumonia & $60(39.7)$ & $16(37.2)$ & $44(40.7)$ & 0.160 & 1 & 0.689 \\
\hline Cardiopulmonary resuscitation & $12(7.9)$ & $\mathrm{I}(2.3)$ & II (I0.2) & 2.597 & 1 & 0.107 \\
\hline Sepsis & $5(3.3)$ & $\mathrm{I}(2.3)$ & $4(3.7)$ & 0.182 & 1 & 0.669 \\
\hline Seizure & $5(3.3)$ & $0(0.0)$ & $5(4.6)$ & 2.059 & 1 & 0.151 \\
\hline Hypoxic encephalopathy & $5(3.3)$ & $0(0.0)$ & $5(4.7)$ & 2.059 & 1 & 0.151 \\
\hline Gastrointestinal bleeding & $5(3.3)$ & $0(0.0)$ & $5(4.7)$ & 2.059 & 1 & 0.151 \\
\hline Stroke & $\mathrm{I}(0.7)$ & $0(0.0)$ & $\mathrm{I}(0.9)$ & 0.401 & 1 & 0.527 \\
\hline Acute myocardial infarction & $\mathrm{I}(0.7)$ & $\mathrm{I}(2.3)$ & $0(0.0)$ & 2.528 & 1 & 0.112 \\
\hline Multiple organ failure & I (0.7) & $\mathrm{I}(2.3)$ & $0(0.0)$ & 2.528 & 1 & 0.112 \\
\hline Hospitalization duration, day (mean \pm SD) & $18.6 \pm 15.2$ & $19.5 \pm 18.9$ & $18.2 \pm 13.5$ & & & 0.625 \\
\hline Follow-up at our psychiatric outpatient clinic after discharge & $75(49.7)$ & $25(58.1)$ & $50(46.3)$ & 1.726 & 1 & 0.189 \\
\hline $\begin{array}{l}\text { Follow-up duration at our psychiatric outpatient clinic after } \\
\text { discharge, day (mean } \pm S D \text { ) }\end{array}$ & $998.7 \pm|56| .4$ & $780.4 \pm 1470.9$ & $1103.3 \pm 1607.5$ & & & 0.419 \\
\hline
\end{tabular}

lower, it may be bias that we only recruited survived patients in our study. American research failed to show net-benefit of banning organophosphates and carbamates through risk-trading off analysis ${ }^{19}$ because even the acute toxicity may decrease, effect of change on diet may be hard to be estimated. There was no significant difference between repeaters and nonrepeaters regarding which pesticide they chose, even the trend observed in serum $\mathrm{AChE}$ level may suggest organophosphate was chosen more in nonrepeaters; our patients might not choose their suicide toxin specifically since they possibly could not tell the difference in toxicity between these three pesticides. Nevertheless, further study may be warranted to know their choosing process, in order to make patient-centred plan about suicide prevention.

In this study group, most patients were married (72\%). This proportion is higher than that of our previous study $(52.9 \%),{ }^{13}$ which may be due to some group differences; for example, our patients lived mostly in rural areas, where more people are married. There was statistical significance regarding marriage between repeaters and nonrepeaters $(\mathrm{p}=0.001)$, repeaters were less likely to be married
(53.5\%), they were more likely to be single, divorced or widow. This corresponds to previous systemic review about suicide repetition by suicide means other than pesticide. $^{2}$ As indicated by a previous study, divorce was a risk factor for suicide death, ${ }^{20}$ and the opposite may be true for marriage - it may be a protective factor. In a Tunisian study, ${ }^{21}$ it was revealed that a higher percentage of repeaters were divorced or separated compared to nonrepeaters. A study that focused on cultural differences stated that Asian women may not benefit from marriage as Western females do, ${ }^{22}$ but support from their partner still played an important role to repeaters in our study. One previous study demonstrated gender and age differences in that divorced males showed a stronger effect size for suicide propensity, while females who were never married, aged below 35 or older than 65 had lower suicide odds. ${ }^{20}$ Due to the small sample size, gender and age subgroups were not analyzed in our group of repeaters. For patients not married, psychosocial support is indicated.

Thirty-eight percent of our patients had previously used psychiatric services, mostly in the outpatient department. Lin et $\mathrm{al}^{23}$ studied 468 Taiwanese patients who 
Table 4 Laboratory Findings of Patients with Pesticide Poisoning, Stratified into Two Subgroups According to Suicide History as Repeaters and Nonrepeaters $(n=151)$

\begin{tabular}{|c|c|c|c|c|}
\hline \multirow[t]{2}{*}{ Variables } & All Patients $(n=151)$ & Repeaters $(n=43)$ & Nonrepeaters $(n=108)$ & P-value \\
\hline & n (\%) & n (\%) & n (\%) & \\
\hline Serum acetylcholinesterase level (nadir), U/L (mean $\pm S D)$ & $2226.0 \pm 3675.2$ & $3058.6 \pm 5 \mid 40.2$ & $1897.8 \pm 2870.2$ & 0.087 \\
\hline \multicolumn{5}{|l|}{ Vital signs (emergency department) } \\
\hline Glasgow coma scale score (mean \pm SD) & $11.3 \pm 4.6$ & $10.9 \pm 4.8$ & II $.4 \pm 4.6$ & $0.50 \mathrm{I}$ \\
\hline Body temperature, $C($ mean $\pm S D)$ & $36.1 \pm 1.1$ & $36.2 \pm 1.0$ & $36.0 \pm 1.1$ & 0.326 \\
\hline Pulse rate, time/min (mean $\pm S D)$ & $97.4 \pm 21.7$ & $101.9 \pm 21.0$ & $95.6 \pm 21.9$ & 0.119 \\
\hline Respiratory rate, time/min (mean $\pm \mathrm{SD}$ ) & $19.8 \pm 4.0$ & $20.6 \pm 4.2$ & $19.5 \pm 3.9$ & 0.160 \\
\hline Systolic blood pressure, $\mathrm{mmHg}($ mean $\pm \mathrm{SD})$ & $|45.4 \pm 3| .2$ & $138.9 \pm 22.5$ & $148.1 \pm 33.9$ & 0.117 \\
\hline Diastolic blood pressure, $\mathrm{mmHg}($ mean $\pm \mathrm{SD})$ & $84.3 \pm 19.4$ & $83.7 \pm 18.4$ & $84.5 \pm 19.9$ & 0.815 \\
\hline Hemoglobin, g/dL (mean $\pm S D)$ & $13.9 \pm 2.3$ & $13.8 \pm 2.6$ & $13.9 \pm 1.1$ & 0.771 \\
\hline White blood cell, $1000 / \mu \mathrm{L}($ mean $\pm S D)$ & $14.2 \pm 7.1$ & $14.8 \pm 7.6$ & $14.0 \pm 6.9$ & 0.536 \\
\hline Segment, \% (mean \pm SD) & $76.7 \pm 14.3$ & $76.8 \pm 16.0$ & $76.7 \pm 13.7$ & 0.972 \\
\hline Platelets, $1000 / \mu \mathrm{L}($ mean $\pm S D)$ & $243.7 \pm 87.6$ & $245.0 \pm 104.9$ & $243.1 \pm 80.4$ & 0.905 \\
\hline Blood urea nitrogen, $\mathrm{mg} / \mathrm{dL}($ mean $\pm \mathrm{SD})$ & $18.5 \pm 14.0$ & $16.0 \pm 9.2$ & $19.4 \pm 15.3$ & 0.251 \\
\hline Creatinine, $\mathrm{mg} / \mathrm{dL}($ mean $\pm \mathrm{SD})$ & $1.98 \pm 7.43$ & $1.03 \pm 0.38$ & $2.35 \pm 8.74$ & 0.331 \\
\hline Potassium, mEq/L (mean \pm SD) & $3.7 \pm 0.7$ & $3.6 \pm 0.7$ & $3.7 \pm 0.7$ & 0.493 \\
\hline Sodium, mEq/L (mean \pm SD) & $140.3 \pm 4.1$ & $140.1 \pm 3.4$ & $140.4 \pm 4.3$ & 0.657 \\
\hline Alanine aminotransferase, $\mathrm{U} / \mathrm{L}($ mean $\pm \mathrm{SD})$ & $45.4 \pm 56.2$ & $44.6 \pm 41.5$ & $45.7 \pm 60.9$ & 0.919 \\
\hline \multicolumn{5}{|l|}{ Arterial blood gas } \\
\hline $\mathrm{pH}($ mean $\pm \mathrm{SD})$ & $7.312 \pm 0.149$ & $7.307 \pm 0.130$ & $7.314 \pm 0.156$ & 0.792 \\
\hline Partial pressure of carbon dioxide, $\mathrm{mmHg}($ mean $\pm \mathrm{SD})$ & $43.7 \pm 6.6$ & $43.3 \pm 4.7$ & $43.8 \pm 17.4$ & 0.879 \\
\hline Partial pressure of oxygen, $\mathrm{mmHg}($ mean $\pm \mathrm{SD})$ & $125.2 \pm 109.0$ & $114.2 \pm 90.9$ & $129.8 \pm \mid 15.7$ & 0.465 \\
\hline Bicarbonate, $\mathrm{mmol} / \mathrm{L}(\operatorname{mean} \pm \mathrm{SD})$ & $21.3 \pm 5.6$ & $20.3 \pm 4.6$ & $21.7 \pm 5.9$ & 0.202 \\
\hline Base deficit, mEq/L (mean $\pm S D)$ & $-4.4 \pm 6.3$ & $-5.6 \pm 5.6$ & $-4.0 \pm 6.6$ & 0.200 \\
\hline
\end{tabular}

visited the ER for suicidal behavior and found that 53.6\% had previous psychiatric intervention. The lower proportion found in our study group might be because the patient group in Lin's study came from a metropolitan area where more psychiatric services are available. The follow-up rate in our psychiatric outpatient department was $49.7 \%$, while only $26.1 \%$ of patients visited the outpatient department after discharge in Lin's study, ${ }^{23}$ which may be due to our study protocol only including patients who received a psychiatric consultation; therefore, a higher follow-up

Table 5 Prediction of Suicide Repetition Using Logistic Regression Analysis $(n=|5|)$

\begin{tabular}{|c|c|c|c|c|c|c|c|}
\hline \multicolumn{4}{|c|}{ Univariate Analysis } & \multicolumn{4}{|c|}{ Multivariate Analysis } \\
\hline Variable & $\begin{array}{l}\text { Odds } \\
\text { Ratio }\end{array}$ & $\begin{array}{l}95 \% \text { Confidence } \\
\text { Interval }\end{array}$ & p value & Variable & $\begin{array}{l}\text { Odds } \\
\text { Ratio }\end{array}$ & $\begin{array}{l}\text { 95\% Confidence } \\
\text { Interval }\end{array}$ & p value \\
\hline Marital status & 3.399 & I.589-7.272 & $0.002 * *$ & Marital status & 2.916 & |.234-6.89| & $0.015 *$ \\
\hline $\begin{array}{l}\text { Past psychiatric } \\
\text { service usage }\end{array}$ & 7.752 & $3.497-|7.24|$ & $0.000 * * *$ & $\begin{array}{l}\text { Past psychiatric } \\
\text { service usage }\end{array}$ & 6.897 & $3.012-15.625$ & $0.000 * * *$ \\
\hline Complex suicide & $2.48 I$ & I.188-5.208 & $0.016^{*}$ & Complex suicide & 1.689 & $0.725-3.937$ & 0.224 \\
\hline
\end{tabular}

Notes: ${ }^{*}<0.05, * * p<0.01,{ }^{* * *} p<0.001$. 
Table 6 Psychiatric Comorbidities of Patients with Pesticide Poisoning, Stratified into Two Subgroups According to Suicide History as Repeaters and Nonrepeaters $(n=|5|)$

\begin{tabular}{|c|c|c|c|c|c|c|}
\hline \multirow[t]{2}{*}{ Variables } & All Patients $(n=|5|)$ & Repeaters $(n=43)$ & Nonrepeaters $(n=\mid 08)$ & & & \\
\hline & n (\%) & n (\%) & n (\%) & $x^{2}$ & df & P-value \\
\hline Adjustment disorder & $23(15.2)$ & $3(7.0)$ & $20(18.5)$ & 3.173 & I & 0.075 \\
\hline Depressive disorder & $101(66.9)$ & $30(69.8)$ & 7I (65.7) & 0.225 & I & 0.635 \\
\hline Bipolar disorder & $2(1.3)$ & I (2.3) & I (0.9) & 0.461 & I & 0.497 \\
\hline Psychotic disorder & $16(10.6)$ & $7(16.3)$ & $9(8.3)$ & 2.050 & I & 0.152 \\
\hline Substance use disorder & $46(30.5)$ & $16(37.2)$ & $30(27.8)$ & 1.291 & I & 0.256 \\
\hline Alcohol use disorder & $39(25.8)$ & $15(34.9)$ & $24(22.2)$ & 2.574 & I & 0.109 \\
\hline Delirium or dementia & $9(6.0)$ & $\mathrm{I}(2.3)$ & $8(7.4)$ & 1.417 & I & 0.234 \\
\hline Deferred & $5(3.3)$ & I (2.3) & $4(3.7)$ & 0.182 & I & 0.669 \\
\hline
\end{tabular}

rate was expected. As many as $72.1 \%$ of repeaters had previously used psychiatric services $(\mathrm{p}<0.001)$; since they had previously attempted suicide, they might have been exposed to psychiatric resources earlier than nonrepeaters, including an ER psychiatric consultation or being referred by another specialty to a psychiatric outpatient, psychotherapy or psychiatric ward admission. Lin's study also revealed that the number of psychiatric admissions of repeaters was more than that of nonrepeaters, ${ }^{23}$ which may indicate that repeaters presented a more severe psychopathology. Repeaters had a trend of shorter follow-up duration in our psychiatric outpatient department compared to nonrepeaters $(\mathrm{p}=0.419)$, which might be because a higher proportion of repeaters tend to have more cluster B personality traits, ${ }^{24}$ which makes it hard for them to maintain a therapeutic relationship. If the need of suicide repeaters can be discovered and ensured earlier when they encounter psychiatric service, repeated suicide attempts might be preventable.

The rate of our patients having a psychiatric diagnosis was $96.7 \%$, this could be due to the selection bias of our study, since our inclusion criteria required a psychiatric consultation or outpatient department record. The most presented diagnosis was depressive disorder (66.9\%), which included major depressive disorder, dysthymic disorder and unspecified depressive disorder. The role of depression in suicide has been well studied, including feelings of hopelessness, ${ }^{25}$ a high comorbidity of anxiety and agitation that escalates the suicide risk, ${ }^{26}$ and the undertreatment of depression contributing to suicidal behavior. Another important statistic is substance use disorder, which reached $30.5 \%$ and was within a previous study range of $19-63 \%{ }^{27}$ The interaction and comorbidity of mood disorders and substance use disorders should be considered, ${ }^{28}$ however, for psychiatric comorbidities, there were no significant differences between repeaters and nonrepeaters, although some trends could be observed, including adjustment disorder (repeaters 7.0\%, nonrepeaters $18.5 \%$ ) and alcohol use disorder (repeaters 34.9\%, nonrepeaters $22.2 \%$ ). For the trend observed in regard to adjustment disorder, it can be suggested that nonrepeaters attempted suicide in response to an external stress, while for repeaters, it was more likely to occur in the context of ongoing psychiatric illness. This reiterates the need for person-centred care. That is, if a person presents with a suicide attempt there is a need to ensure that people receive relevant support to deal with adjustment issues and to manage psychiatric illness; as later attempts could reflect that treatment did not adequately address the original co-morbid issues. Regarding alcohol use disorder, repeaters in our study had a prevalence rate $(34.9 \%)$ that was two-fold of an aforementioned German study, ${ }^{29}$ which may be race or culture difference, further study may be warranted for explanation.

This study had three limitations in different areas. First, we conducted a retrospective study that relied on medical records for analysis; no structured diagnostic interviews were performed, and the reliability of the information was thus limited. A longitudinal, observational study may be better, since it would provide more reliable information 
and we could learn more about each case. Furthermore, if we had full accessibility to the National Health Database, we could trace the patients for a longer period of time, since patients in our study were lost to follow-up after discharge, yet they may have used another psychiatric service. Second, this study only focused on the case series of one medical center, which was an inadequate sample size, additionally, the patient was mostly transferred from local clinic or regional hospital, the generalisability was lacking. Third, the follow-up duration should be longer; although $90 \%$ of suicide reattempts happen within the first 2 years of follow-up, ${ }^{30}$ and our mean length of follow-up was 2.73 years, longer follow-up may still obtain more information. Finally, this study is also limited by lacking data for patients without a psychiatric consultation and lacking data between 2016 and 2019.

\section{Conclusion}

As suicide repeaters comprised $28.5 \%$ of our pesticide cohort patients, early identification of clinical and psychiatric characteristics, including marital status, psychiatric service usage, is emphasized. This helps ensuring that the needs of people who have made a suicide attempt are addressed, it reduces the likelihood of suicide repetition. Further studies are warranted.

\section{Disclosure}

The authors report no conflicts of interest in this work.

\section{References}

1. Large M, Kaneson M, Myles N, Myles H, Gunaratne P, Ryan C. Metaanalysis of longitudinal cohort studies of suicide risk assessment among psychiatric patients: heterogeneity in results and lack of improvement over time. PLoS One. 2016;11(6):e0156322. doi:10.1371/journal.pone. 0156322

2. Mendez-Bustos P, de Leon-martinez V, Miret M, Baca-Garcia E, Lopez-Castroman J. Suicide reattempters: a systematic review. Harv Rev Psychiatry. 2013;21(6):281-295. doi:10.1097/HRP.000000000 0000001

3. Oh SH, Park KN, Jeong SH, Kim HJ, Lee CC. Deliberate self-poisoning: factors associated with recurrent self-poisoning. $\mathrm{Am}$ J Emerg Med. 2011;29(8):908-912. doi:10.1016/j.ajem.2011.03.015

4. Monnin J, Thiemard E, Vandel P, et al. Sociodemographic and psychopathological risk factors in repeated suicide attempts: gender differences in a prospective study. $J$ Affect Disord. 2012;136(1-2):35-43. doi:10.1016/j.jad.2011.09.001

5. Mew EJ, Padmanathan P, Konradsen F, et al. The global burden of fatal self-poisoning with pesticides 2006-15: systematic review. J Affect Disord. 2017;219:93-104. doi:10.1016/j.jad.2017.05.002

6. Gunnell D, Knipe D, Chang SS, et al. Prevention of suicide with regulations aimed at restricting access to highly hazardous pesticides: a systematic review of the international evidence. Lancet Glob Health. 2017;5(10):e1026-1037. doi:10.1016/S2214-109X(17)30299-1
7. Lin TJ, Walter FG, Hung DZ, et al. Epidemiology of organophosphate pesticide poisoning in Taiwan. Clin Toxicol. 2008;46 (9):794-801. doi:10.1080/15563650801986695

8. Noshad H, Ansarin K, Ardalan MR, Ghaffari AR, Safa J, Nezami N. Respiratory failure in organophosphate insecticide poisoning. Saudi Med J. 2007;28(3):405-407.

9. Sahin HA, Sahin I, Arabaci F. Sociodemographic factors in organophosphate poisonings: a prospective study. Hum Exp Toxicol. 2003;22(7):349-353.

10. Carroll R, Metcalfe C, Gunnell D. Hospital presenting self-harm and risk of fatal and non-fatal repetition: systematic review and meta-analysis. PLoS One. 2014;9(2):e89944.

11. Pushpakumara P, Thennakoon SUB, Rajapakse TN, Abeysinghe R, Dawson AH. A prospective study of repetition of self-harm following deliberate self-poisoning in rural Sri Lanka. PLoS One. 2019;14(2): e0199486. doi:10.1371/journal.pone.0199486

12. Aghanwa HS. The characteristics of suicide attempters admitted to the main general hospital in Fiji Islands. $J$ Psychosom Res. 2000;49 (6):439-445. doi:10.1016/S0022-3999(00)00193-8

13. Lin C, Yen TH, Juang YY, Lin JL, Lee SH. Psychiatric comorbidity and its impact on mortality in patients who attempted suicide by paraquat poisoning during 2000-2010. PLoS One. 2014;9(11): e112160. doi:10.1371/journal.pone. 0112160

14. Liu HF, Ku CH, Chang SS, et al. Outcome of patients with chlorpyrifos intoxication. Hum Exp Toxicol. 2020:960327120920911.

15. Lee CP, Yen TH, Juang YY, Lin C, Lin JL, Lee SH. Psychiatric diagnoses and gender differences in patients admitted for pesticide suicide: an 11-year retrospective study in a general hospital in Taiwan. Taiwan J Psychiatry. 2017;31(2):140-149+iii.

16. Toro K, Pollak S. Complex suicide versus complicated suicide. Forensic Sci Int. 2009;184(1-3):6-9. doi:10.1016/j.forsciint.2008.10.020

17. Liu SH, Lin JL, Weng CH, et al. Heart rate-corrected QT interval helps predict mortality after intentional organophosphate poisoning. PLoS One. 2012;7(5):e36576. doi:10.1371/journal.pone.0036576

18. Sungur M, Guven M. Intensive care management of organophosphate insecticide poisoning. Crit Care. 2001;5(4):211-215. doi:10.1186/cc1025

19. Gray GM, Hammitt JK. Risk/risk trade-offs in pesticide regulation: an exploratory analysis of the public health effects of a ban on organophosphate and carbamate pesticides. Risk Anal. 2000;20 (5):665-680. doi:10.1111/0272-4332.205060

20. Yeh JY, Xirasagar S, Liu TC, Li CY, Lin HC. Does marital status predict the odds of suicidal death in taiwan? A seven-year population-based study. Suicide Life Threat Behav. 2008;38 (3):302-310. doi:10.1521/suli.2008.38.3.302

21. Mechri A, Mrad A, Ajmi F, et al. Repeat suicide attempts: characteristics of repeaters versus first-time attempters admitted in the emergency of a Tunisian general hospital. L'Encephale. 2005;31(1 Pt 1):65-71. doi:10.1016/S0013-7006(05)82373-5

22. Chen YY, Wu KC, Yousuf S, Yip PS. Suicide in Asia: opportunities and challenges. Epidemiol Rev. 2012;34:129-144. doi:10.1093/ epirev/mxr025

23. Lin CJ, Lu HC, Sun FJ, Fang CK, Wu SI, Liu SI. The characteristics, management, and aftercare of patients with suicide attempts who attended the emergency department of a general hospital in northern Taiwan. J Chin Med Assoc. 2014;77(6):317-324. doi:10.1016/j.jcma.2014.02.014

24. Blasco-Fontecilla H, Artieda-Urrutia P, Berenguer-Elias N, et al. Are major repeater patients addicted to suicidal behavior? Adicciones. 2014;26(4):321-333. doi:10.20882/adicciones.38

25. Minkoff K, Bergman E, Beck AT, Beck R. Hopelessness, depression, and attempted suicide. Am J Psychiatry. 1973;130(4):455-459. doi:10.1176/ajp.130.4.455

26. Angst J, Angst F, Stassen HH. Suicide risk in patients with major depressive disorder. J Clin Psychiatry. 1999;60 Suppl 2:57-62;discussion 75-56, 113-116.

27. Schneider B. Substance use disorders and risk for completed suicide. Arch Suicide Res. 2009;13(4):303-316. doi:10.1080/13811110903263191 
28. Tondo L, Baldessarini RJ, Hennen J, et al. Suicide attempts in major affective disorder patients with comorbid substance use disorders. $J$ Clin Psychiatry. 1999;60:113-116.

29. Boenisch S, Bramesfeld A, Mergl R, et al. The role of alcohol use disorder and alcohol consumption in suicide attempts-a secondary analysis of 1921 suicide attempts. Eur Psychiatry. 2010;25 (7):414-420. doi:10.1016/j.eurpsy.2009.11.007
30. Parra-Uribe I, Blasco-Fontecilla H, Garcia-Pares G, et al. Risk of re-attempts and suicide death after a suicide attempt: a survival analysis. BMC Psychiatry. 2017;17(1):163. doi:10.1186/s12888-017-1317-z

\section{Publish your work in this journal}

Neuropsychiatric Disease and Treatment is an international, peerreviewed journal of clinical therapeutics and pharmacology focusing on concise rapid reporting of clinical or pre-clinical studies on a range of neuropsychiatric and neurological disorders. This journal is indexed on PubMed Central, the 'PsycINFO' database and CAS, and is the official journal of The International Neuropsychiatric Association (INA). The manuscript management system is completely online and includes a very quick and fair peer-review system, which is all easy to use. Visit http://www.dovepress.com/testimonials.php to read real quotes from published authors. 